\title{
Extrapleural Pneumonectomy for Sarcoma of the Lung in a Pediatric Patient
}

\author{
Michael Schweigert ${ }^{1}$ (1) Ana B. Almeida ${ }^{1}$ Jessica Pablik ${ }^{2}$ \\ ${ }^{1}$ Department of Surgery, University Hospital Schleswig-Holstein, \\ Luebeck, Germany \\ ${ }^{2}$ Department of Pathology, University Hospital, Dresden, Germany \\ Address for correspondence Prof. Dr. Michael Schweigert, \\ Department of Surgery, University Hospital Schleswig-Holstein, \\ Ratzeburger Allee 160, 23538 Luebeck, Germany \\ Thorac Cardiovasc Surg Rep 2022;11:e20-e22. \\ (e-mail: Michael.Schweigert@uksh.de).
}

\begin{abstract}
Keywords

- pediatric sarcoma

- pediatric thoracic surgery

- extrapleural pneumonectomy

Pediatric sarcomas are rare entities. Bone sarcomas and rhabdomyosarcoma are most common, whereas primary sarcoma of the lung is extremely uncommon. Pneumonectomy is only very infrequently performed in the pediatric population. We report on a 16-year-old girl with an undifferentiated high-grade pleomorphic sarcoma of the left lung. There was no comorbidity and no history of previous malignant disease. The huge tumor originated from the left lower lobe and had spread to the left pleura. As part of a multimodal approach, she underwent extrapleural pneumonectomy with en bloc resection of the pleura, lung, pericardium, and diaphragm.
\end{abstract}

\section{Introduction}

Pneumonectomy in children is very infrequently performed. ${ }^{1}$ In developed countries, the most common indications are congenital anomalies and primary or metastatic pulmonary tumors. ${ }^{1,2}$ Just a handful of cases of extrapleural pneumonectomy (EPP) in children have been reported. ${ }^{2}$ Pediatric sarcomas represent only $13 \%$ of all pediatric malignancies and soft tissue sarcoma is even more uncommon. ${ }^{3}$

In this report, we present the case of a 16-year-old girl who underwent EPP for pulmonary sarcoma as part of a multimodal treatment concept.

\section{Case Description}

A 16-year-old girl with no previous illnesses started to complain about pain in the left shoulder and the left chest wall. When the pain did not subside, she consulted her general physician who diagnosed shoulder-arm syndrome and prescribed a pain gel. She did not get better and started to feel unwell and tired. A little later she started coughing. At that time, she was referred to a pediatric unit at a local hospital. Chest X-ray showed a large opacity in the lower half of the left chest (-Fig. 1A). She was diagnosed with pneumonia and received antibiotics. When no improvement occurred, computed tomography (CT) was performed. The CT scan showed a large tumor of the left lower pulmonary lobe with spread to the pleura. She was now transferred to the pediatric department at a German university hospital.

Whole body magnetic resonance imaging (MRI) revealed no distant metastatic disease. Biopsies showed a sarcoma without specific lineage differentiation. Molecular pathology was able to detect ALK-EML4 translocation. Initially, systemic cancer therapy with chemotherapy and crizotinib was started. Following the receipt of the reference pathology, the CWS (Cooperative Soft Tissue Sarcoma Study Group) guidance was sought and polychemotherapy following the VAIA regimen (vincristine, adriamycin, ifosfamide, and actinomycin D) in combination with alectinib was given. After initial response, new tumor progress was seen and the therapy regimen was adapted again. The last chemotherapy included gemcitabine, vinorelbine, and docetaxel together with lorlatinib. Following 6 months of systemic cancer therapy, the multidisciplinary team (MDT) meeting recommended referral to thoracic surgery for possible resection of the tumor. received

July 7, 2021

accepted after revision

November 18, 2021
DOI https://doi.org/ $10.1055 / \mathrm{s}-0042-1742781$ ISSN 2194-7635.

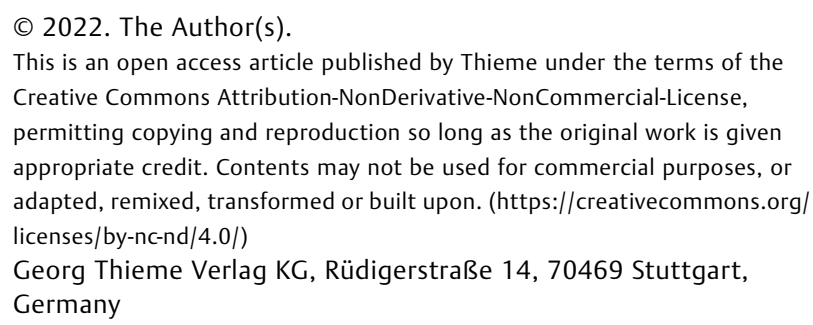



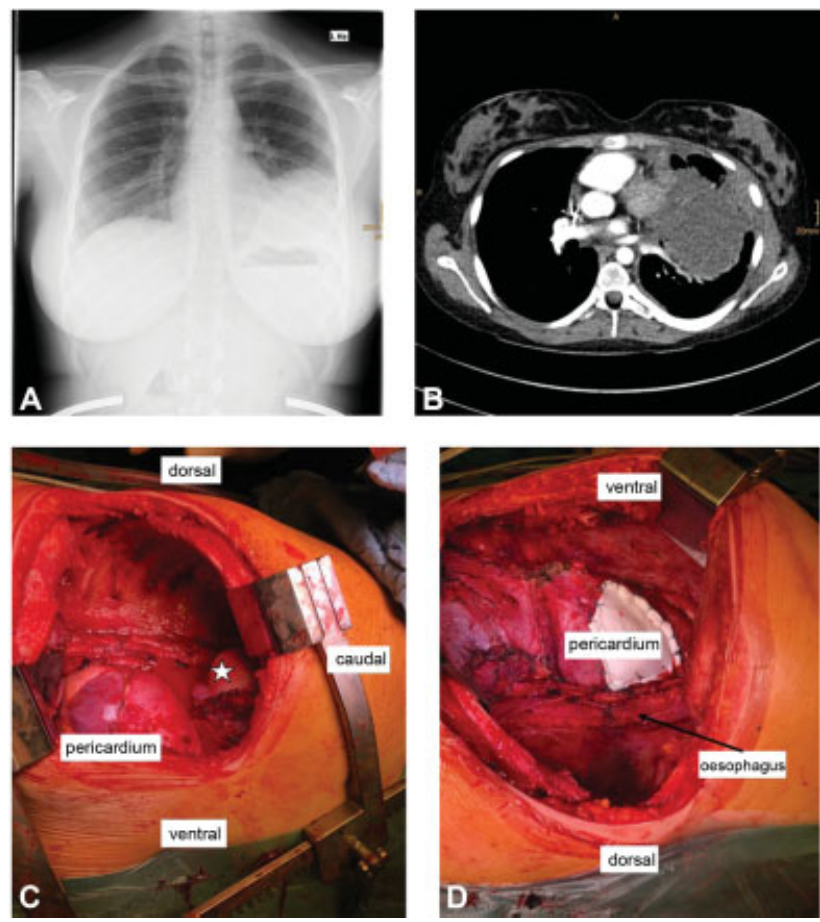

Fig. 1 (A) Initial chest-X-ray. (B) CT scan after 6 months of systemic cancer therapy. (C) Intraoperative situs. Situs after removal of the EPP specimen. Partial resection of the pericardium and the diaphragm (star). (D) Intraoperative situs. Reconstruction of the pericardium. CT, computed tomography; EPP, extrapleural pneumonectomy.

Restaging proofed that the tumor was still limited to the left chest cavity (-Fig. 1B) with no occurrence of distant metastatic disease. Complete macroscopic resection seemed achievable and the patient underwent thoracotomy. Intraoperatively, a large tumorous mass comprising the entire left lung with spread to the pleura but no invasion of the muscular and osseous parts of the chest wall was encountered. Neighboring structures as the oesophagus or the big vessels were not invaded. The parietal pleura could easily be separated from the chest wall and the mediastinum. Extrapleural pneumonectomy with en bloc resection of the left lung, pleura, parts of the diaphragm, and the pericardium was performed (-Fig. 1C). Several frozen sections proofed clear resection margins. The pericardium was reconstructed using Gore-Tex Soft Tissue Patch (W. L. Gore \& Associates, Newark, Delaware, United States; - Fig. 1D).

Pathology showed an undifferentiated pleomorphic sarcoma with spindle cell and epithelioid components (-Fig. 2). There was intratumoral necrosis $(<10 \%)$ and the KI-67 proliferation index was 60 to $70 \%$. The postoperative course was largely uneventful and the patient was discharged home at postoperative day (POD) 14 in good condition and with no need for supplemental oxygen. The MDT meeting suggested further therapy with ALK inhibitor (brigatinib) and adjuvant radiotherapy. The patient and her parents, however, decided against the radiotherapy and close follow-up was started.

\section{Discussion}

Nonrhabdomyosarcoma soft tissue sarcoma (NRSTS) accounts for ca. $4 \%$ of all pediatric malignancies. ${ }^{3}$ Undifferentiated highgrade pleomorphic sarcoma (UHGPS) is even more uncommon in children and comprises only 2 to $6 \%$ of all pediatric sarcomas. ${ }^{4}$ UHGPS is a highly aggressive tumor with considerable both metastatic rate and risk of local recurrence. Scattered aggregates or discrete components of epithelioid cells as in our case have been reported previously in the pediatric population and are considered to be associated with a less favorable prognosis. ${ }^{4}$ The proximal variant of epithelioid sarcoma (PES) constitutes an important differential diagnosis. ${ }^{5}$ PES is a highly aggressive, undifferentiated, soft tissue sarcoma, too. It accounts for approximately 4 to $8 \%$ of all NRSTS. PES is characterized by INI 1 (integrase interactor 1 ) inactivation with absence of INI 1 expression in immunohistochemistry. ${ }^{5}$ In our case, however, INI 1 was not inactivated, so that PES could be ruled out.

Modern treatment for pediatric sarcoma is without question multimodal comprising systemic therapy, surgery, and radiotherapy. $^{3}$ As in adult patients, targeted therapy has moved to the center of interest in pediatric oncology. 6,7 Many current pediatric oncology trials investigate the application of targeted therapy or the combination of
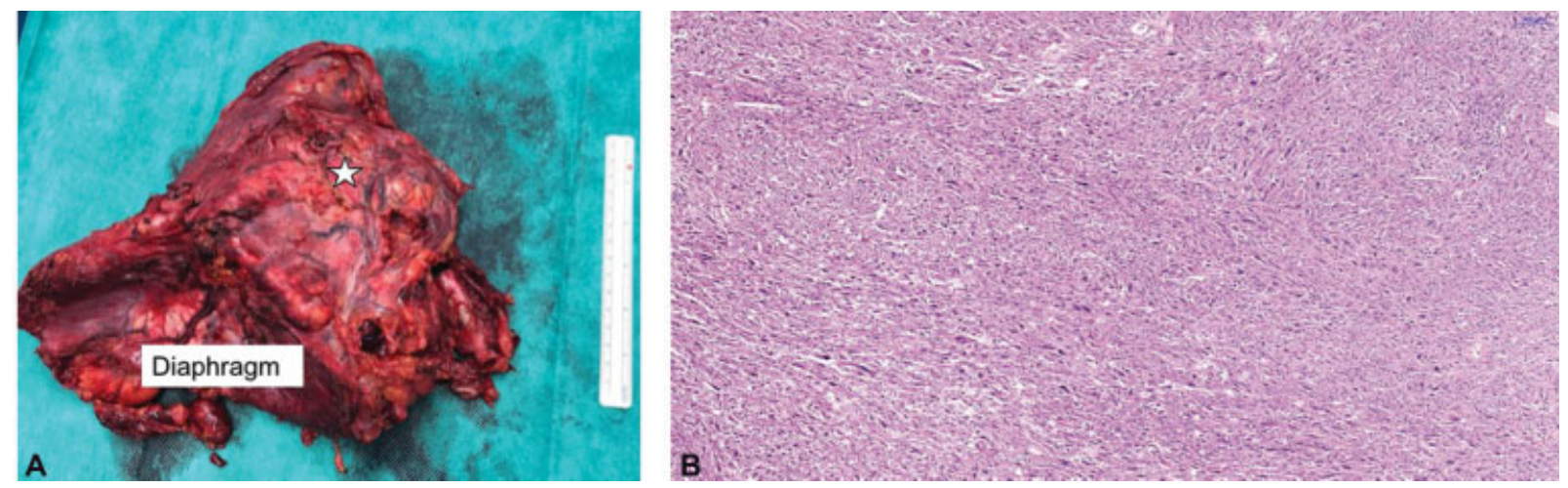

Fig. 2 (A) Specimen. EPP-specimen with pericardium (star), parietal pleura und diaphragm. (B) Histology. HE staining ( $\times 10$ magnification) showing an epithelioid, pleomorphic neoplasia with some spindle cells. EPP, extrapleural pneumonectomy; HE, hematoxylin and eosin. 
e22 Sarcoma of the Lung in a Pediatric Patient Schweigert et al.

conventional chemotherapy with targeted drugs. ${ }^{7}$ The reported case is particularly interesting and innovative because molecular pathology confirmed ALK-EML4 translocation opening up the possibility for targeted therapy with a proteinase inhibitor. ${ }^{8}$ As a consequence, the applied systemic therapy was from the outset combining conventional chemotherapy with targeted drugs as crizotinib.

\section{Conclusion}

In pediatric thoracic surgery, pneumonectomy is very infrequently performed. ${ }^{1,2}$ Indications for EPP in children have only anecdotally been reported. ${ }^{2}$ The treatment of pediatric sarcoma is multimodal with radical surgery being the cornerstone of successful therapy. Rapid progress in molecular pathology, the availability of new substances and the more widespread use of targeted therapy for pediatric malignancies will probably result in better response rates of systemic therapy for pediatric sarcoma. Increased effectiveness of upfront chemotherapy means that it is likely that more patients will eventually be considered for radical surgery. As there currently exists only limited information regarding the feasibility and outcome of EPP in the pediatric population, we feel that it is important to share experience to build up a better and well-founded basis for decision-making in thoracic surgery for pediatric sarcoma. Against this background, we report our case to illustrate that within a multimodal approach, EPP is an option for pediatric sarcoma patients where the tumor is limited to one chest cavity.

\section{Note}

This study was presented at the 29th European Conference on General Thoracic Surgery, June 20-22, 2021 (Virtual ESTS Meeting).

Conflict of Interest

None declared.

\section{References}

1 Blyth DF, Buckels NJ, Sewsunker R, Soni MA. Pneumonectomy in children. Eur J Cardiothorac Surg 2002;22(04):587-594

2 Flores RM, Su W, Lal D, Rusch VW, La Quaglia MP. Extrapleural pneumonectomy in children. J Pediatr Surg 2006;41(10): 1738-1742

3 Loeb DM, Thornton K, Shokek O. Pediatric soft tissue sarcomas. Surg Clin North Am 2008;88(03):615-627, viivii.

4 Alaggio R, Collini P, Randall RL, Barnette P, Million L, Coffin CM. Undifferentiated high-grade pleomorphic sarcomas in children: a clinicopathologic study of 10 cases and review of literature. Pediatr Dev Pathol 2010;13(03):209-217

5 Thway K, Jones RL, Noujaim J, Fisher C. Epithelioid sarcoma: diagnostic features and genetics. Adv Anat Pathol 2016;23(01): 41-49

6 Harwood JL, Alexander JH, Mayerson JL, Scharschmidt TJ. Targeted chemotherapy in bone and soft-tissue sarcoma. Orthop Clin North Am 2015;46(04):587-608

7 Cohen JW, Akshintala S, Kane E, et al. A systematic review of pediatric phase I trials in oncology: toxicity and outcomes in the era of targeted therapies. Oncologist 2020;25(06):532-540

8 Felkai L, Bánusz R, Kovalszky I, et al. The presence of ALK alterations and clinical relevance of crizotinib treatment in pediatric solid tumors. Pathol Oncol Res 2019;25(01):217-224 